\title{
El fútbol como herramienta para el trabajo de los valores y actitudes en la ESO según las Competencias Básicas

\author{
Football as a tool for the job of the values and attitudes in the ESO according to the Basic Competencies
}

\author{
*David Rodríguez Ruiz \\ *Universidad de Las Palmas de Gran Canaria (España)
}

\begin{abstract}
Resumen: Con el presente estudio queremos exponer una experiencia práctica llevada a cabo en el I.E.S. José Arencibia Gil (Telde - Gran Canaria), con alumnos de $3^{\circ}$ de la E.S.O., basado en el desarrollo de las competencias básicas. El objetivo del mismo es trabajar «los valores y las actitudes» desde el marco que nos traza el Decreto 127/2007 del BOC de junio del 2007, utilizando el futbol como herramienta, debido a ese papel de «formación integral» que posé la Educación Física. Para evaluar la consecución de los objetivos se confección una planilla simplificada que parte de las ideas expuestas por Gil et al. (2006) en su propuesta de valores e indicadores a evaluar en los juegos y deportes y para valorar el grado de conciencia y consecución de los objetivos, así como el nivel de implicación en el trabajo se pasó entre el alumnado un cuestionario. Obteniendo como resultado que más del $96 \%$ de los alumnos se divierten, esfuerzan, mantienen unas relaciones sociales coherentes, juegan limpio, se controlan emocionalmente y respetan las normas, por otro lado, del total de los alumnos que componen las clases $(\mathrm{n}=118)$ un 80\% de ellos había entendido el objetivo de las sesiones. 49 alumnos creían que se pretendía «aprender a relacionarse» y 45 a «mejorar las habilidades y destrezas». Los resultados nos llevan a la conclusión de que las competencias básicas «refuerzan y dan mayor importancia al papel integrador de la Educación Física» y que ésta se muestra como «pilar de la enseñanza en valores $y$ actitudes sin descuidar sus contenidos y objetivos especificos».
\end{abstract}

Palabra clave: Valores, Actitudes, Futbol, Competencias Básicas, Educación.

\begin{abstract}
In this study we present a practical experience conducted in the I.E.S. José Arencibia Gil (Telde - Gran Canaria) with students from $3^{\circ} \mathrm{ESO}$, based on the development of basic competencies. The aim is to work «values and attitudes» from the context that traces the BOC Decree 127/2007 of June 2007, using football as a tool, because the role of «integral education» which Physical Education holds. To assess the achievement of the objectives was made for a simplified form of the work presented by Gil et al. (2006) in its proposition of values and indicators to evaluate in games and sports and to assess the level of awareness and achievement of the objectives and the level of involvement in work rose from a student questionnaire. With the result over $96 \%$ of students having fun, strive to maintain a coherent social relationships, play fair, emotionally controlled and respect the rules, on the other hand, that the total number of students included in the classes ( $\mathrm{n}=118)$ an $80 \%$ of them had understood the purpose of the sessions. 49 students thought that it was intended to «learn how to interact» and 45 «to improve the skills and abilities». The results lead us to conclude that the basic competencies «strengthen and give greater importance to the integrative role of Physical Education» and it shows as «pillar of education in values and attitudes without releasing their specifics contents and objectives».
\end{abstract}

Key words: Values, Attitudes, Football, Basic Competencies, Education.

\section{Introducción}

Con la llegada del nuevo sistema educativo, regulado por la Ley Orgánica 2/2006, de 3 de mayo, de educación (LOE), se produce una modificación a nivel ideológico y práctico que se ha plasmado en el REAL DECRETO 1513/2006, de 7 de diciembre, por el que se establecen las enseñanzas mínimas de la Educación Física.

Dicho cambio comienza por la propia definición de currículo, que se entiende, tal y como dice el art. 5, como «El conjunto de objetivos, competencias básicas, contenidos, métodos pedagógicos y criterios de evaluación de esta etapa» (REAL DECRETO 1513/2006, de 7 de diciembre). Se observará que se añade un nuevo elemento a la anterior concepción de currículo: las competencias básicas.

Entendemos por Competencias Básicas, siguiendo el DECRETO 127/2007, «el conjunto de conocimientos, habilidades y actitudes que debe alcanzar el alumnado al finalizar la enseñanza básica para:

Su realización y desarrollo personal.

Ejercer debidamente la ciudadanía.

Incorporarse a la vida adulta.

Seguir aprendiendo a lo largo de la vida».

Desde esta perspectiva las Competencias Básicas supondrían la combinación de «habilidades prácticas, conocimientos, motivaciones, valores éticos, actitudes, emociones y otros componentes sociales que actúan conjuntamente para lograr una acción eficaz»)(MolinayAntolin, 2008, p.83) que se cimentan en la etapa de Educación Primaria para ser

Fecha recepción: 04-06-09 - Fecha envío revisores: 26-06-09 - Fecha de aceptación: 19-12-09 Correspondencia: David Rodríguez Ruiz

Facultad de Ciencias de la Actividad Física y el Deporte Campus universitario de Tafira s/n

35017 Universidad de Las Palmas de Gran Canaria

Email: drodriguez@def.ulpgc.es alcanzadas en la de Educación Secundaria Obligatoria (ESO), donde deben culminar su desarrollo. Con este propósito el trabajo de las Competencias Básicas se asocia a los contenidos en sus diferentes formas, es decir, contenidos conceptuales o saber qué, contenidos procedimentales o saber cómo y contenidos actitudinales o saber ser (Real Decreto 1513/2006 y Real Decreto 1631/2006).

Aunque no se encuentre ninguna Competencia Básica «referida a la competencia corporal y motriz» (Molina y Antolín, 2008, p. 85), diferentes autores opinan que la Educación Física aporta al alumnado, en mayor o menor medida, un desarrollo competencial (Lleixá, 2007; Del Villar, 2007 y 2009; Molina y Antolín, 2008; Vaca, 2008; Buscà y Capllonch, 2008).

En este sentido, a la hora de desarrollar el currículo en Educción Física hay que tener presentes las aportaciones de la Comisión encargada de elaborarlo para la Dirección General de Ordenación e innovación Educativa de la Consejería de Educación del Gobierno de Canarias (mayo 2007). Los cuales nos dicen que Competencia Básica es «la capacidad de responder a demandas complejas y llevar a cabo tareas diversas de forma adecuada y que supone la combinación de:

\section{Habilidades prácticas.}

Conocimientos.

Motivación.

Valores éticos.

Actitudes.

Emociones.

Otros componentes sociales y de comportamiento...

...que se movilizan conjuntamente para lograr una acción eficaz».

Por tanto, vemos que la Educación Física, según nos exponen Gómez Rijo et al (2008): «... trata todos los Objetivos Generales de la Etapa, si bien lo hace en algunos con una especial incidencia en los aspectos didácticos», debido principalmente al mismo concepto de 
«Educación Física como educación integral a través del cuerpo y el movimiento cuya finalidad es el desarrollo personal y social del alumno» (Figura 1).

El objetivo de este trabajo es exponer la experiencia y los resultados obtenidos al utilizar el fútbol como herramienta para el trabajo de los valores y actitudes en la E.S.O. según las Competencias Básicas.

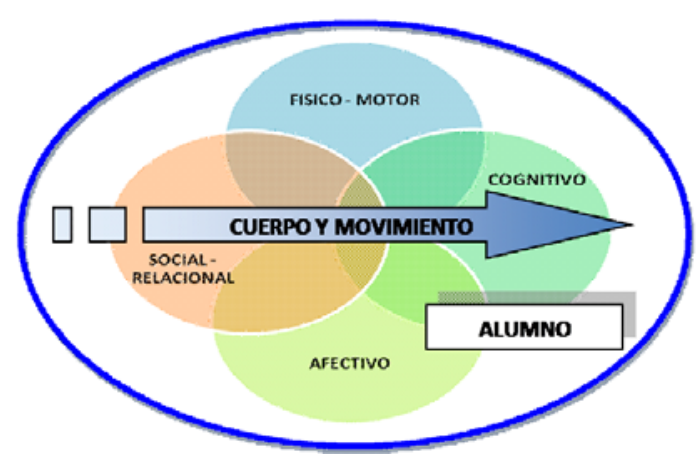

Figura 1: La Educación Física en el desarrollo humano (Adaptado de Gómez Rijo et al. 2008)

\section{Contextualización}

Debido a ese papel de formación integral que posé la Educación Física se eligió esta área para intentar trabajar y desarrollar los valores y las actitudes en $3^{\circ}$ de la E.S.O. en el I.E.S. JoséArencibia Gil, ya que los equipos educativos de los distintos grupos comprobamos una serie de faltas en sus relaciones personales:

\section{Relación entre alumnos.}

Integración de alumnos recién llegados al centro.

Respeto a las actividades en grupo.

Se optó por el futbol como herramienta de trabajo debido a que es una actividad aceptada por el alumnado, si bien el fin no es el dominio del juego en sí mismo sino potenciar los objetivos de relación, integración y respeto. Esta elección responde a las características propias de las actividades físicas colectivas o deportivas, donde en la mayor parte de los casos es necesaria la colaboración entre los integrantes de los equipos para alcanzar obetivos comunes. Para lo cual el alumnado «debe aprender a aceptar y practicar normas sociales y a comportarse de forma responsable y solidaria» (Lleixà, 2007 p.34).

La experiencia no se basa únicamente en centrar las sesiones de la unidad didáctica en la consecución de dichos valores y actitudes, sino que através de los contenidos específicos necesarios para alcanzar los objetivos de área, poder desarrollar las competencias precisas para mantener unas relaciones sociales correctas. Esta decisión responde a la misma naturaleza de la educación física, donde «el aprendizaje del respeto y la aceptación de las reglas en los juegos y actividades deportivas tiene mucho que ver con la comprensión de los códigos de conducta para la convivencia» (Lleixà, 2007 p.34).

El centro cuenta con una serie de peculiaridades a tener en cuenta, como son:

- Cancha deportiva preparada para poder realizar la práctica del futbol-sala.

- Material suficiente para llevar a cabo la actividad: balones de futbol-sala, balones de goma espuma y conos.

Las clases tienen la particularidad de:

- Clases de 55 minutos que como hay que darles 5 minutos para cambiarse antes y después de la sesión. El tiempo real de la clase es de 45 minutos.

- La estructura de la clase queda de la siguiente forma: 2 minutos de planteamiento de la clase (objetivos y organización), 5 minutos de calentamiento, la parte principal que consta de 30 minutos aproximadamente y 5 minutos de vuelta a la calma, para terminar la sesión con una reflexión final de 2 minutos y el aseo.

\section{Planteamiento didáctico}

\subsection{Contenidos}

Las distintas sesiones se desarrollaron atendiendo al bloque de contenidos, a los criterios de evaluación y las competencias básicas que debemos trabajar según nos marca la ley educativa:

- LEY ORGÁNICAEDUCATIVA(L.O.E.) 2/2006, de 3 de mayo, de Educación. B.O.E. núm. 106. Jueves 4 mayo 2006.

- REAL DECRETO 1513/2006, de 7 de diciembre, por el que se establecen las enseñanzas mínimas de la Educación Física. B.O.E. núm. 293. Viernes 8 diciembre 2006.

- DECRETO 127/2007, 25 de mayo, por el que se establece la ordenación y el currículo de la Educación Secundaria Obligatoria en la ComunidadAutónoma de Canarias. B.O.C. núm. 113. Jueves 7 de junio de 2007.

Los «bloques de contenidos» que abordamos estaban incluidos en la unidad didáctica número 5 de la programación de $3^{\circ}$ de la E.S.O., que englobó a los deportes de equipo y más específicamente sobre el futbol, desde el punto de vista «conceptual, procedimental, actitudinal y transversal» (Tabla 1).

\begin{tabular}{|c|c|}
\hline Conceptuales & $\begin{array}{l}\text { 1.Conocimiento de las habilidades básicas necesarias para jugar al fútbol. } \\
\text { 2.Conocimiento de los aspectos técnicos básicos del fútbol. } \\
\text { 3. Conocimiento de los principios básicos de ataque y defensa. } \\
\text { 4.Conocimiento de las principales reglas de juego. }\end{array}$ \\
\hline Procedimentales & 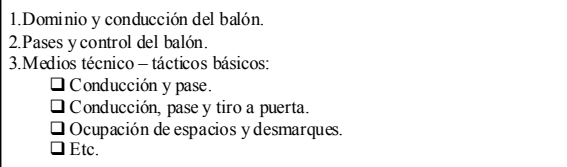 \\
\hline Actitudinales & $\begin{array}{l}\text { 1.Participación activa. } \\
\text { 2.Colaborar para conseguir un objet ivo común. } \\
\text { 3.Respeto por las reglas de juego. }\end{array}$ \\
\hline Transversales & $\begin{array}{l}\text { 1.Integrar a todos en la realización de tácticas para la consecución de los } \\
\text { objetivos. } \\
\text { 2. Respetar las diferencias en el dominio de habilidades. }\end{array}$ \\
\hline
\end{tabular}

Hemos añadido el bloque de contenidos «transversales» siguiendo las pautas elaboradas por el profesor Martínez Navarro (2008), pues creemos que refleja claramente contenidos específicos de valores y actitudes. Por otro lado, aclarar el énfasis en la palabra «todos», ya que la utilizamos conscientemente para referimos al alumnado en general: de ambos sexos y además a las diferencias de nivel de dominio motriz, motivación y con posibles discapacidades.

\subsection{Relación de los Contenidos con el desarrollo de las} Competencias Básicas

Mediante la realización de los contenidos enunciados anteriormente (tabla 1) se intentaba que los alumnos tuvieran la posibilidad de desarrollar el nivel competencial propio del nivel educativo, para lo cual se pone énfasis en que el alumnado consiguiera los siguientes objetivos por competencia:

\section{Comunicación lingüística:}

a. Emisión y comprensión de mensajes, vocabulario específico.

b. Uso correcto en las consignas con las que se presentan las tareas y se interviene en las actividades, etc ...

\section{Matemática:}

a. Secuencia de acciones, formas, medidas, trayectorias espaciales...

b. Dominio de los espacios y cálculos de trayectorias, etc...

Conocimiento e interacción con el mundo fisico:

a. Adecuada percepción de uno mismo y del espacio físico en el que nos movemos.

b. La habilidad para interactuar conél: moverse en él, orientarsey resolver problemas en los que intervengan los objetos y su posición, etc... 


\section{Socialyciudadana:}

a. Trabajo en grupo, responsabilidades, aprender a convivir, desde la elaboración y aceptación de las reglas, desde el respeto a la autonomía personal, la participación y la valoración de la diversidad.

b. Las actividades dirigidas a la adquisición de las habilidades motrices requieren la capacidad de asumir las diferencias así como las posibilidades y limitaciones propias y ajenas.

c. El cumplimiento de la norma en el juego colabora en la aceptación de códigos de conducta para la convivencia.

d. Las actividades físicas competitivas pueden generar conflictos en los que es necesaria la negociación, basada en el diálogo, como medio de resolución, etc...

Culturaly artística:

a. Favorece un acercamiento al fenómeno deportivo como espectáculo mediante el análisis y la reflexión crítica ante la violencia en el deporte $u$ otras situaciones contrarias a la dignidad humana que en él se producen, así como ante las actitudes positivas que puede mostrar (compañerismo, superación, sacrificio...), etc...

Aprender a aprender:

a. Diseño, aplicación y valoración de sus propias tareas motrices, lo que implica iniciar al alumnado en el aprendizaje y ser capaz de continuarlo de manera autónoma.

b. Requiere ser consciente de lo que se sabe, de las propias posibilidades y limitaciones, como punto de partida del aprendizaje motor, desarrollando un repertorio motriz variado que facilite su transferencia a tareas más complejas, etc...

Autonomía e iniciativa personal:

a. Desarrollo del esquema corporal, de las habilidades perceptivo - motrices y de las coordinaciones que permitan desenvolverse óptimamente en su entorno; también comprometiéndonos con una metodología activa, reflexiva y participativa que fomente la confianza en uno mismo, la responsabilidad, la autocrítica, la toma de decisiones con progresiva autonomía y la capacidad de superación.

b. Se reforzará la iniciativa personal y una sana valoración del rendimiento y del éxito que promuevan el esfuerzo y la superación, sin entrar en conflicto con la debida prioridad que el mismo esfuerzo, el disfrute y las relaciones interpersonales deben tener sobre el resultado del juego., etc...

\section{Digitaly sobre el tratamiento de la información:}

a. Desarrollo de la habilidad de buscar información referente al tema a tratar, tanto en internet como en la prensa diaria, para posteriormente reflexionar sobre su contenido y aplicación a los valores que queremos trabajar.

\subsection{Sesiones}

El problema de educar en función de las competencias es enunciado por Zabala y Arnau (2007, p. 44): «Enseñar competencias implica saber intervenir en situaciones reales que, por ser reales, siempre son complejas». Por este motivo, los juegos y los deportes, en este caso el

Tabla 2: Sesión Inicial de "futbol", de la Unidad Didáctica "Deportes de Equipo"

\begin{tabular}{|c|c|}
\hline \multicolumn{2}{|r|}{ Sesión Inicial } \\
\hline Actividades Iniciales & $\begin{array}{l}\text { - Futbolín: jugar al futbol pero cogidos de la mano y haciendo } \\
\text { filas enfrentadas, al igual que un futbolín, que se pueden } \\
\text { desplazar nada más que de forma lateral en una línea (balón de } \\
\text { gomaespuma). ( } 7 \text { min) }\end{array}$ \\
\hline Actividades de Desarrollo & $\begin{array}{l}\text { - Futbol por parejas: Jugar una partido de futbol pero por parejas, } \\
\text { cogidos de la mano (balón de gomaespuma). ( } 7 \mathrm{~min}) \\
\text { - Chicos contra Chicas: Los chicos cogidos de la mano, por } \\
\text { parejas, y las chicas libre (balón de gomaespuma). ( } 7 \mathrm{~min} \text { ) }\end{array}$ \\
\hline Actividades Finales & $\begin{array}{l}\text { - Partidos en dos canchas reducidas, arbitrados por un alumno. } \\
\text { Dos equipos por cancha. A marcar dos goles o cambio de } \\
\text { enfrentamientos a los } 5 \mathrm{~min} \text {. (14 min) }\end{array}$ \\
\hline $\begin{array}{l}\text { Criterios de Evaluación } \\
\text { especificos }\end{array}$ & $\begin{array}{l}\text { - Respeto a las normas. } \\
\text { - Relación con los demás. } \\
\text { - Colaboración con los compañeros. } \\
\text { - Datos del Cuestionario de evaluación de actitudes y valores. }\end{array}$ \\
\hline
\end{tabular}

fútbol, presentan situaciones propicias para el desarrollo de las competencias desde el punto de vista de la complejidad y el del carácter procedimental de la Educación Física. En consecuencia, el desarrollo de las Competencias Básicas se alcanza mediante el planteamiento de contenidos (tabla 1) que vayan implicando al alumnado a resolver una serie de situaciones distintas y complejas «que constituyen una actuación competente desde un pensamiento complejo» (Zabalay Arnau, 2007, p. 43).

Para ir introduciendo a los alumnos en situaciones complejas que planteen resolución de problemas, no sólo procedimentales sino también actitudinales se planteó una progresión de sesiones, de las cuales tan sólo vamos a desarrollar la parte principal (Tabla 2 a 5).

Tabla 3: Sesión de desarrollo (1) de "futbol", de la Unidad Didáctica "Deportes de Equipo"

\begin{tabular}{|c|l|}
\hline \multicolumn{2}{|c|}{ Sesión Desarrollo (l) } \\
\hline Actividades Iniciales & $\begin{array}{l}\text { - Organizados en filas, conducción del balón a través de los conos } \\
\text { realizando zig-zag: alternando las piernas, sólo con la derecha, sólo } \\
\text { con la izquierda,... (5 min) } \\
\text { - Misma forma de organización, pasar el balón al compañero de la } \\
\text { otra fila entre cada espacio delimitado por los conos. (5min) } \\
\text { - Idem al anterior pero acabando con tiro a puerta. (5 min) }\end{array}$ \\
\hline Actividades de Desarrollo & $\begin{array}{l}\text { - Organizados en filas, pase a un compañero, que está fijo y es el que } \\
\text { expone el ejercicio, que devuelve el balón y tiro a puerta del que } \\
\text { inicia el movimiento. (7 min) }\end{array}$ \\
\hline Actividades Finales & $\begin{array}{l}\text { - Partidos en dos canchas reducidas, arbitrados por un alumno. Dos } \\
\text { equipos por cancha. A marcar dos goles o cambio de enfrentamientos } \\
\text { a los 5 min. (13 min) }\end{array}$ \\
\hline especificos & $\begin{array}{l}\text { - Exposición de los ejercicios por parte de algunos alumnos } \\
\text { (excogidos por el profesor). } \\
\text { - Expresión en público. } \\
\text { - Respeto al compañero que expone. } \\
\text { - Datos del Cuestionario de evaluación de actitudes y valores. }\end{array}$ \\
\hline
\end{tabular}

Tabla 4: Sesión de desarrollo (2) de "futbol", de la Unidad Didáctica "Deportes de Equipo"

\begin{tabular}{|c|c|}
\hline \multicolumn{2}{|r|}{ Sesión Desarrollo (2) } \\
\hline Actividades Iniciales & $\begin{array}{l}\text { - Rondos: Organización por grupos, en espacios reducidos, } \\
\text { formando un círculo y uno se la queda, en el centro. Tiene que } \\
\text { intentar interceptar algún pase de los compañeros. ( } 3 \mathrm{~min}) \\
\text { - Idem con sólo control y pase por los que están fuera. }(3 \mathrm{~min}) \\
\text { - Idem al anterior pero quedándose dos alumnos } / \text { as. ( } 3 \mathrm{~min}) \\
\text { - Idem al anterior pero quedándose tres alumnos } / \text { as. (3 min) }\end{array}$ \\
\hline Actividades de Desarrollo & $\begin{array}{l}\text { - Partido en cancha reducida, aprovechando los grupos de los } \\
\text { "rondos". (6 min) } \\
\text { - Ampliar la cancha cruzando los grupos de los "rondos". ( } 6 \mathrm{~min})\end{array}$ \\
\hline Actividades Finales & $\begin{array}{l}\text { - Partido normal, arbitrado por un alumno. Equipo fuera y entra a } \\
\text { jugar cuando alguno de los otros dos equipos marque dos goles o se } \\
\text { cumplan los cinco minutos, quedándose el equipo que vaya } \\
\text { ganando. }(11 \mathrm{~min})\end{array}$ \\
\hline $\begin{array}{c}\text { Criterios de Evaluación } \\
\text { especificas }\end{array}$ & $\begin{array}{l}\text { - Control del pase. } \\
\text { - Dominio espacial. } \\
\text { - Respeto a las normas. } \\
\text { - Relación con los demás. } \\
\text { - Colaboración con los compañeros. } \\
\text { - Datos del Cuestionario de evaluación de actitudes y valores. }\end{array}$ \\
\hline
\end{tabular}

Tabla 5: Sesión de final de "futbol", de la Unidad Didáctica "Deportes de Equipo"

\begin{tabular}{|c|c|}
\hline \multicolumn{2}{|r|}{ Sesión Final } \\
\hline Actividades Iniciales & $\begin{array}{l}\text {-Partido de futbol, con las reglas normales y en dos canchas } \\
\text { reducidas y dos equipos por cancha. ( } 5 \mathrm{~min})\end{array}$ \\
\hline $\begin{array}{c}\text { Actividades de Desarrollo y } \\
\text { Finales }\end{array}$ & $\begin{array}{l}\text {-Partido de futbol, sólo se puede dar dos toques al balón y } \\
\text { luego es obligado pasara an compañero/a. ( } 5 \text { min) } \\
\text {-ídem, pero hay que buscar a un compañero/a de sexo } \\
\text { contrario. ( } 5 \text { min) } \\
\text {-ídem, pero sólo se puede meter gol dentro del área marcada } \\
\text { ( } 7 \text { min) } \\
\text { - Ídem, pero sólo se puede meter gol cuando todo el equipo esté } \\
\text { en el campo contrario. ( } 8 \text { min) }\end{array}$ \\
\hline $\begin{array}{l}\text { Criterios de Evaluación } \\
\text { especificos }\end{array}$ & $\begin{array}{l}\text { - Control del pase. } \\
\text { - Dominio espacial. } \\
\text { - Respeto a las normas. } \\
\text { - Relación con los demás. } \\
\text { - Colaboración con los compañeros. } \\
\text {-Datos del Cuestionario de evaluación de actitudes y valores. }\end{array}$ \\
\hline
\end{tabular}




\subsection{Evaluación}

El control de la realización de los contenidos por parte del alumnado, para una posterior evaluación, fue registrada por el profesor en una planilla simplificada de recogida de datos, mendiante la valoración de positivo o negativo. La confección de dicha planilla parte de las ideas expuestas por Gil et al. (2006) en su propuesta de valores e indicadores a evaluar en los juegos y deportes (diversión, esfuerzo, relaciones sociales, juego limpio, control emocional, respeto a las normas). De todos los valores e indicadores que nos proponen los autores para poder evaluar los juegos y deportes, hemos seleccionado los que consideramos más representativos y fáciles de emitir un juicio rápido de cada alumno o alumna de la clase:

Participa y se divierte (Diversión)

Aporta su esfuerzo y destreza para ayudar al grupo (Esfuerzo) Mantiene el orden y la armonía del grupo independientemente del resultado (Relaciones sociales y Control emocional).

- Discrimina a los compañeros por razones de competencia motriz, raza o sexo (Relaciones sociales).

Comete faltas con el objetivo de obtener un beneficio (Juego limpio).

Controla sus reacciones ante situaciones que le son adversas (Control emocional).

- Valora y acepta las normas como parte del juego (Respeto a las normas).

La elección de estos indicadores, a parte de favorecer la recogida de datos, responde a la visión del fútbol como actividad motriz colectiva, que exige a los alumnos a un trabajo en equipo, que favorece el desarrollo de la competencia de aprender a prender que fevorece el propio aprendizaje de los valores y actitudes necesarios para las relaciones sociales. Hecho que refuerza el desarrollo de la competencia social y ciudadana mediante el fomento de la integración, el respeto, la cooperación y la igualdad (Lleixà, 2007; Molina y Antolín, 2008).

Además creemos que los indicadores nos aproximan a conseguir los criterios de evaluación que nos marca la LOE para $3^{\circ}$ de la ESO, en función del desarrollo de las Competencias Básicas:

Ajustar con eficiencia las habilidades motrices especificas a distintas situaciones psicomotrices y sociomotrices, considerando su lógica en la resolución de problemas motores, reconociendo aspectos básicos de su estructura y valorando críticamente los fenómenos socioculturales que se manifiestan en ellas, aceptando el nivel de ejecución alcanzado.

Valorar una actitud de responsabilidad individual y colectiva en la práctica de actividades fisico-motrices por encima de la búsqueda desmedida de los resultados, manifestando conductas solidarias y cooperativas dentro de una labor de equipo para la consecución de objetivos comunes.

Para evaluar el grado de conciencia y consecución de los objetivos, así como el nivel de implicación en el trabajo, se pasó un cuestionario entre los alumnos con diferentes items (tabla 7).

Tabla 6: Cuestionario de evaluación del grado de concienciación de los alumnos (marcar una respuesta por pregunta)

\begin{tabular}{|c|c|}
\hline $\begin{array}{l}\text { ¿QUÉ OBJETIVO TENÍA LA } \\
\text { CLASE?: }\end{array}$ & $\begin{array}{l}\text { वAPRENDER A JUGAR AL FUTBOL. } \\
\text { वMEJORAR LAS HABIIDADES Y DESTREZAS. } \\
\text { पAPRENDER A RELACIONARSE. } \\
\text { DRESPETAR LAS NORMAS Y A LOS COMPAÑEROS. }\end{array}$ \\
\hline $\begin{array}{l}\text { LAS ACTIVIDADES TE } \\
\text { PARECIERON: }\end{array}$ & $\begin{array}{l}\text { DDIVERTIDAS. } \\
\text { DABURRIDAS. }\end{array}$ \\
\hline CREES QUE ERAN: & $\begin{array}{l}\text { DFACILES. } \\
\text { DDIFÍCILES. }\end{array}$ \\
\hline $\begin{array}{l}\text { LAS INDICACIONES DEL } \\
\text { PROFESOR ERAN: }\end{array}$ & $\begin{array}{l}\text { घUN ROLLO. } \\
\text { aNO LAS ENTENDÍA. } \\
\text { aSUFICIENTES. } \\
\text { aBUENAS. }\end{array}$ \\
\hline $\begin{array}{l}\text { EL PROFESOR HACÍA LAS } \\
\text { ACTIVIDADES: }\end{array}$ & $\begin{array}{l}\text { DPARA TODOS. } \\
\text { वPARA LOS QUE NO SABEN JUGAR AL FUTBOL. } \\
\text { UPARA LOS QUE SABEN JUGAR AL FUTBOL. }\end{array}$ \\
\hline
\end{tabular}

La primera pregunta es la que directamente nos va a dar el entendimiento de las sesiones por parte del alumnado. En cambio, la segunday tercera recaban la información necesaria para evaluar el interés que las tareas despiertan en los mismos. El cuestionario finaliza con dos preguntas donde se intenta obtener la percepción de los alumnos sobre la calidad de las indicaciones del profesor y el planteamiento de las actividades.

\section{Resultados}

Tras recoger los datos de las sesiones en las planillas simplificadas de criterios e indicadores se obtuvieron los siguientes resultados de valoración del profesor (positiva o negativa)

Tabla 7: Resultados obtenidos tras la observación del profesor de los criterios e indicadore elegidos para la planilla simplificada

\begin{tabular}{|l|l|}
\hline \multicolumn{1}{|c|}{ INDICADORES (CRITERIOS) } & \multicolumn{1}{c|}{ RESULTADOS } \\
\hline Participa y se divierte (Diversión) & $100 \%$ positiva \\
\hline $\begin{array}{l}\text { Aporta su esfuerzo y destreza para ayudar al grupo } \\
\text { (Esfuerzo) }\end{array}$ & $\begin{array}{l}96,6 \%(\mathrm{n}=114) \text { de forma positiva y un 3,4\% } \\
(\mathrm{n}=4) \text { de forma negativa }\end{array}$ \\
\hline $\begin{array}{l}\text { Mantiene el orden y la armonía del grupo } \\
\text { independientemente del resultado (Relaciones } \\
\text { sociales y Control emocional). }\end{array}$ & $\begin{array}{l}98,3 \%(\mathrm{n}=116) \text { de forma positiva y un } 1,7 \% \\
(\mathrm{n}=2) \text { de forma negativa. }\end{array}$ \\
\hline $\begin{array}{l}\text { Discrimina a los compañeros por razones de } \\
\text { competencia motriz, raza o sexo (Relaciones } \\
\text { sociales). }\end{array}$ & $\begin{array}{l}99,2 \%(\mathrm{n}=117) \text { de forma positiva y un } 0,8 \% \\
(\mathrm{n}=1) \text { de forma negativa. }\end{array}$ \\
\hline $\begin{array}{l}\text { Comete faltas con el objetivo de obtener un } \\
\text { beneficio (Juego limpio). }\end{array}$ & $100 \%$ positiva \\
\hline $\begin{array}{l}\text { Controla sus reacciones ante situaciones que le son } \\
\text { adversas (Control emocional). }\end{array}$ & $\begin{array}{l}98,3 \%(\mathrm{n}=116) \text { de forma positiva y un } 1,7 \% \\
(\mathrm{n}=2) \text { de forma negativa }\end{array}$ \\
\hline $\begin{array}{l}\text { Valora y acepta las normas como parte del juego } \\
\text { (Respeto a las normas). }\end{array}$ & $100 \%$ positiva \\
\hline
\end{tabular}

Para evaluar el grado aceptación y concienciación del alumnado, se recogieron las respuestas elegidas por los alumnos que conforman la muestra de nuestro trabajo obteniendo los siguientes resultados para cada uno de los items empleados en el cuestionario:

Respecto a la primera pregunta, «¿quéobjetivo tenía la clase?», del total de los alumnos que componen las clases ( $\mathrm{n}=118$ ), un $80 \%$ de ellos había entendidos el proposito de las sesiones, un $42 \%$ de los alumnos creían que se pretendía «aprender a relacionarse» y un 38\% pensaba que estaban encaminadas a «mejorar las habilidades y destrezas» (figura 2).

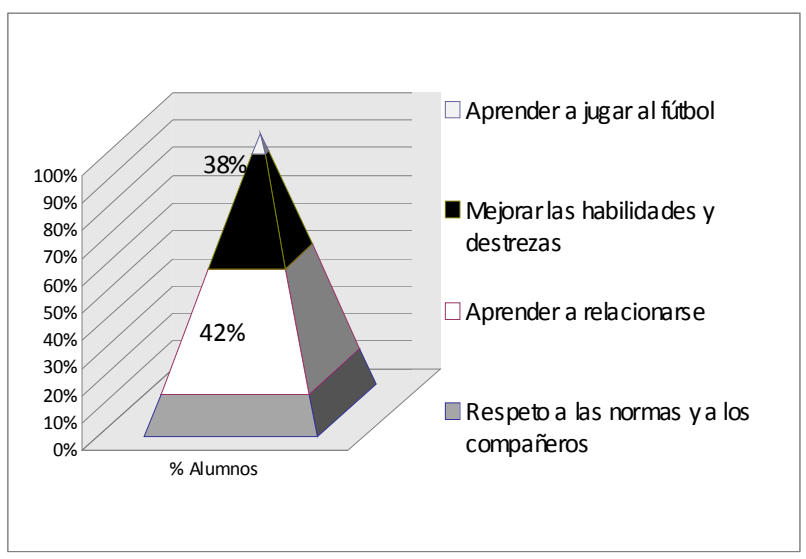

Figura 2: Porcentaje de alumnos que eligen los diferentes “objetivos".

Es decir, 49 alumnos creían que se pretendía «aprender a relacionarse» y 45 a «mejorar las habilidades y destrezas》. Por otro lado, 16 de ellos hacen referencia a que se pretendía trabajar el «respeto a las normas y a los compañeros» y por último, tan sólo 8 de los alumnos de la muestra se decantaban por elegir la opción de «Aprender a jugar al futbol». Donde todos los alumnos encuestados (100\%) considera las actividades «fáciles de realizar, divertidas y que están destinadas a todos», indiferente a la diferencia de sexo, nivel de dominio, motivación y con posibles discapacidades. 


\section{Discusión}

Al ser la Educación Física un área que intenta producir interaciones, tanto socio-afectivas como motrices y que éstas son mayores que en cualquier otra área (Gil et al., 2006), Es por lo que se optó por ésta para intentar solucionar los problemas planteados en los equipos educativos de los cursos de $3^{\circ}$ de laESO: falta de relación entre alumnos, deintegración de los recién llegados al centro y de respeto a las actividades en grupo.

Análizando la bibliografía especializada encontramos que diferentes autores opinan que la Educación Física aporta al alumnado, en mayor o menor medida, un desarrollo competencial (Lleixá, 2007; Del Villar, 2007 y 2009; Molina y Antolín, 2008; Vaca, 2008; Buscà y Capllonch, 2008). Esta posibilidad parte de la naturaleza propia de las actividades físicas y deportivas que presentan la resolución de problemas en situaciones reales de relación social (Zabala y Arnau, 2007; Ruiz y Cabrera, 2008) y por tanto, favorecen el desarrollo de las Competencias Básicas. A pesar de esta evidencia, muchos autores, como Del Villar (2007 y 2009) y Lleixà (2007) denuncian la ausencia de una Competencia propia para laEducación Física, como podría ser la Competencia Corporal o Motriz y, manifiestan su contradicción al «papel subsidiario y servil como medio para conseguir otras competencias» (Del Villar, 2009, p. 66) que le dá el Real Decreto 1631/2006: «La materia de educación fisica contribuye de manera clara a la consecución de dos competencias básicas: la competencia en el conocimiento y la interacción con el mundo fisico y la competencia social y ciudadana».

En nuestro caso, hemos utilizado éste área para solucionar una problemática que urgía en el centro y a los equipos educativos. Pero, en todo momento, se tuvo en consideración la progamación y los contenidos específicos de la asignatura para la consecución de objetivos de la materia y de la etapa y, tan sólo, se adaptaron dos de los criterios de evaluación que nos marca la LOE para poder centrarnos en el desarrollo y evaluación de las actitudes y valores.

Para la evaluación de la consecución de objetivos se utilizaron una planilla simplificada de registro y un cuestionario para la valoración de la comprensión y aceptación de las tareas por parte de los alumnos. La bibliografía especializada enuncia que los profesores utilizamos frecuentemente en el control del proceso de enseñanza los test, las pruebas, las escalas de observación, las listas de control, las hojas de registro, etc... Estos instrumentos se centran sobre todo en los contenidos procedimentales y a veces, en los conceptuales, «dejando a la experiencia del profesor el control de aspectos actitudinales» (Ortega et la, 2009, p. 22).

En este sentido, la planilla simplificada, elavorada a partir del trabajo de Gil et al (2006), nos ayudó a evaluar la relación e integración en la actividad por parte del alumnado, intentando de esta forma poner rigurosidad en los parámetros de observación. Aún así, creemos que es la parte de la experiencia que debe ser revisada y mejorada para posteriores trabajos, ya que no es fácil observar en las sesiones a tantos alumnos y tantos criterios e indicadores al mismo tiempo. Se intentaron mitigar dichos problemas mediante la elección de indicadores de actuación positiva o negativa, para hacerla más sencilla y fácil de cumplimentar. Con el objetivo de tener unos criterios de evaluación claros y que se basaran en las relaciones interpersonales y no sólo en las capacidades de realización de las mismas y por último, poder valorar la capacidad de reflexión y diálogo de todo los ocurrido en la sesión. Aun así, creemos que este punto debe marcar una futura línea de investigación y desarrollo de la misma, pues representó el punto más difícil de concretar en nuestro trabajo.

Por otro lado, se utilizó el cuestionario para saber la implicación del alumnado en las tareas propuestas. La bibliografía nos señala que es el instrumento más utilizado por los docentes para saber las inquietudes de los alumnos, pero en la mayoría de los casos puede existir poca rigurosidad o no registran los que se pretende medir (Silverman y Subramanian, 1999; Thomas y Nelson, 2007; Ortega et al, 2009). En nuestro caso, nos decidimos por este instrumento para comprobar el grado de concienciación del alumnado hacia las tareas y encontramos que el $80 \%$ de los alumnos tenían claro que los objetivos de las actividades eran «aprender a relacionarse» $(\mathrm{n}=49)$ y «mejorar las habilidades $y$ destrezas» $(\mathrm{n}=45)$. Por tanto, podemos ver que las sesiones cumplían los objetivos que los equipos educativos se planteaban mejorar y por otro lado, los objetivos específicos del área.

Se eligió el futbol como herramienta de trabajo debido a que es una actividad aceptada por los alumnos/as. Pero esto, que podría resultar una ventaja para trabajar una serie de aspectos como la integración, las relaciones interpersonales, podría tornarse negativo, sin la orientación necesaria por parte del docente, «haciendo que afloren actitudes negativas referidas al sectarismo o a la exaltación personal» (Gil et al., 2006, p. 157). Hay que entender que el deporte por sí mismo no educa, sino que «la trasmisión de valores mediante la práctica deportiva, el desarrollo de valores personales y sociales debe hacerse mediante un planteamiento que considere las características y circunstancias de los alumnos, las del contexto, y las de los agentes implicados en el mismo, de forma que se generen unas directrices precisas quefomenten ese gran potencial? (Ruiz y Cabrera, 2004, p. 18) que tiene el deporte.

En este sentido, hay que destacar el resultado de las preguntas que tratan sobre la predisposición y actitud del profesor y que enuncian un $100 \%$ de acuerdo en decir que las indicaciones eran «buenas» y que las actividades estaban planteadas para «todos» y no sólo para los que sabían jugar al futbol o para los que no sabían.

\section{Conclusiones}

Los resultados nos llevan a la conclusión de que las competencias Básicas «refuerzan y dan mayor importancia al papel integrador de la Educación Física» y que ésta se muestra como «pilar de la enseñanza en valores y actitudes sin descuidar sus contenidos y objetivos especificos».

\section{Referencias bibliográficas}

Buscà, F \& Capllonch, M (2008). De la competencias básicas a las competencia transversales. Aportaciones desde el ámbito de la educación física. Tándem. Didáctica de la Educación Física, 26: 34-51.

DECRETO 127/2007, 25 de mayo, por el que se establece la ordenación y el currículo de la Educación Secundaria Obligatoria en la Comunidad Autónoma de Canarias. B.O.C. núm. 113.Jueves 7 de junio de 2007.

Del Villar, F. (2007). Educar o enseñar. El papel del profesor de educación fisica en el contexto de la nueva LOE. Libro de Actas del VII Congreso Internacional sobre la enseñanza de la educación física y el deporte escolar. Diputación de Badajoz.

Del Villar, F. (2009). La calidad del aprendizaje en educación física. Una propuesta para la educación secundaria Tándem. Didáctica de la Educación Física. 29: 64-77.

Gil, P., López, A., Sánchez-Ajofrin, R.J., Hotelano, M., Codina, P., Zamorano, D., Sánchez, J.A., García, L., Paterna, A., Calero, F.C., Pardo, E., García G. \& Fernández, M. (2006). Educar en valores a través de la práctica en juegos y deportes: eleancia en el talante, valores y actitudes asociados al deporte. Idea La Mancha. Revista de Educación de Castilla-La Mancha, 3: 152-159.

Gómez, A., Díez LJ J, Fernández, J.M., Gorrín, A., Pacheco, JJ \& \& Sosa, G (2008). Nueva propuesta curricular para el área de Educación Física en la Educación primaria. Revista Internacional de Medicina y Ciencias de la Actividad Física y el Deporte vol. 8 (29): 93-108.

Lleixà, T. (2007). Educación Física y competencias básicas: contribución del área a la adquisición de las competencias básicas del curiculo. Tándem. Didáctica de la Educación Física, 23: 31-37.

LEY ORGÁNICA 2/2006, de 3 de mayo, de Educación. B.O.E. núm. 106. Jueves 4 mayo 2006.

Martinez, F. (2008). Elaboración de programaciones y unidades didácticas partiendo de las competencias básicas. Documentación del Centro del Profesorado de Telde. 29 de marzo a 13 de mayo del 2008.

Molina, J.P. \& Antolín, L. (2008). Las competencias básicas en educación física: Una valoración crítica. Ciencia, Cultura y Deporte, nº, vol. 3: $81-86$.

Ortega, E., Calderon, A., Palao, J.M. \& Puigcerver, M.C. (2009). Diseño y validación de un cuestionario para evaluar la actitud percibida del profesor en clase y de un cuestionario para evaluar los contenidos actitudinales de los alumnos durante las clases de educación física en secundaria. Retos. Nuevas tendencias en Educación Fiscia, deporte y Recreación. 4: 22-19.

Real Decreto 1513/2006, de 7 de diciembre, por el que se establecen las enseñanzas mínimas de la Educación Primaria. B OE n 293, de 4 de diciembre, 43053-43102.

Real Decreto 1631/2006, de 29 de diciembre, por el que se establecen las enseñanzas mínimas de la Educación Secundaria Obligatoria. B OE nº 5, de 5 de enero, 677-773.

Ruiz, G., Cabrera, D. (2004). Los valores en el deporte. Revista de Educación. 335: 9-19.

Silverman, S. \& Subramaman, P.R. (1999). Student attitude toward education and physical activity. A review of measurement issues and outcomes. Journal of Teaching in Physical Education. 19: 97-125.

Thomas, R. \& Nelson, J. (2007). Métodos de investigación en actividad física. Ed. Paidotribo. Barcelona.

Vaca, M. (2008). Contribución de la Educación Física escolar a las competencias básicas señaladas en la LOE para la educación primaria. Tándem. Didáctica de la Educación Física, 26: 52-61. 